Biochem. J., 127: 531 (1972).

25. Wieland, $\mathrm{O}: \mathrm{L}(+)$ Lactate determination with lactate dehydrogenase from yeast In: H. U. Bergmeyer: Methods of Enzymatic Analysis, p. 271 (Academic Press, New York, 1965)

26. Wrenshall, G. A.: The kinetics of isotope turnover in mammalian systems. Can.

J. Biochem. Physiol., 33: 909 (1955).
27. We thank the Canadian Medical Research Council (Grant MRC MA5473) and the Weston Foundation for financial support.

28. Requests for reprints should be addressed to: B. H. Robinson, M.D., Research Institute, The Hospital for Sick Children, 555 University Ave., Toronto, Ontario M5G IX8 (Canada).

29. Accepted for publication May 4, 1976

\title{
The Adaptation of the Fetal Red Cells of Newborn Lambs to Extrauterine Life: The Role of 2,3-Diphosphoglycerate and Adult Hemoglobin
}

\author{
H. BARD, (12) J.-C. FOURON, A. M. GROTHE, M. A. SOUKINI, AND A. CORNET \\ Perinatal Service and Research Center of Hôpital Sainte-Justine, Department of Pediatrics, University of Montreal, \\ Montreal, Quebec, Canada
}

\section{Extract}

The purpose of this study was to determine the interrelationship of the rise and fall of 2,3-diphosphoglycerate (DPG) with the increase in adult hemoglobin and the decrease in red cell oxygen hemoglobin affinity after birth in normal lambs. It was found that the mean maximum DPG level was $26.71 \pm 4.98 \mathrm{~mol} / \mathrm{g} \mathrm{Hb}$ at $7.5 \pm$ 1.1 days. At the same time the mean $P_{50}$ and adult hemoglobin level was $27.0 \neq 1.4 \mathrm{~mm} \mathrm{Hg}$ and $31.1 \pm 11.1 \%$, respectively. In the individual lambs, the level of their maximum DPG correlated inversely with the amount of adult hemoglobin $(r-\mathbf{0 . 7 7}, P<0.05)$. Once the DPG began to decrease, there was an inverse correlation between the DPG and the adult hemoglobin present in the red cell $(r=0.68, P<0.001)$. It appeared that the rise in DPG postanatally is only a compensatory mechanism until an adequate amount of adult hemoglobin is present. This fact was borne out by the second part of the study in which exchange transfusions with adult red cells were performed on five newborn lambs during the first $24 \mathrm{hr}$ after birth and aborted the rise in DPG.

\section{Speculation}

The adaptation from the in utero to the extrauterine environment requires a rapid reduction in red cell oxygen affinity. The decrease in oxygen affinity is achieved by a transitory postnatal increase in intraerythrocyte DPG.

During in utero life the role of high affinity fetal blood is to provide a physiologic advantage that facilitates oxygen transport across the placenta. After birth a more efficient extraction of oxygen by the tissues is needed which is accomplished by the lowered affinity of adult blood.

It was Battaglia et al. (1) who demonstrated in newborn lambs that $\mathrm{pH}$ across the red cell and DPG concentration within the red cell both increase as there is a marked decrease in oxygen affinity compared with fetal blood. Similar postnatal changes in human red cells, although much less pronounced, have been described by Versmold et al. (6).
In order to further the understanding of the great transitory increase in DPG during the first weeks of life in the newborn lamb, the following studies were planned: $(l)$ to follow sequentially in the same animal the rise and fall of DPG, the percentage of adult and fetal hemoglobin along with changes of the oxygen dissociation curve; and (2) to repeat the above study after having provided the newborn animal with an adult oxygen hemoglobin dissociation curve within hours after birth.

\section{MATERIALS AND METHODS}

Mixed breed newborn lambs (Dorset and Suffolk) were used in this study. The experiment was divided up into two sections. The first part was to sample postnatally at 3- to 5 day intervals via the jugular vein normal newborn lambs and to correlate their erythrocyte DPG, $\mathrm{P}_{50}$, and proportion of adult and fetal hemoglobin with postnatal age.

Erythrocyte DPG concentration was determined on fresh heparinized blood according to the method of Keitt (5) and expressed as micromoles per $\mathrm{g} \mathrm{Hb}$. The reagents were obtained from Sigma Chemical Company (7).

The $\mathrm{P}_{50}$ was determined by gas mixing tonometry using a IL 217 blood gas laboratory (8) (213 blood gas analyzer, 237 tonometer, 208 gas mixing system, and 182 co-oximeter). The $\mathrm{P}_{50}$ values were

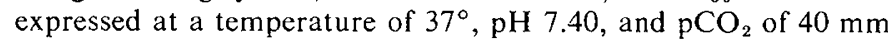
$\mathrm{Hg}$.

Samples of fresh blood were equilibrated in a tonometer for 30 min at $37^{\circ}$ with gas mixtures containing $40 \mathrm{~mm} \mathrm{Hg}( \pm 0.05 \mathrm{~mm}$ $\mathrm{Hg}) \mathrm{CO}_{2}$ and varying proportions of $\mathrm{O}_{2}$ and $\mathrm{N}_{2}$. The tonometer permitted an equilibration and sampling of successive aliquots of blood at different $\mathrm{O}_{2}$ tensons. Measurement of $\mathrm{O}_{2}$ saturation, $\mathrm{pH}$, and $\mathrm{PO}_{2}$ provided the information for plotting the $\mathrm{O}_{2}$ dissociation curve and $\mathrm{P}_{50} . \mathrm{PO}_{2}$ was always converted to $\mathrm{pH} 7.4$ using a Bohr effect of 0.46 (4).

The oxygen saturation obtained by use of the co-oximeter was checked by determining the oxygen contents of blood samples equilibrated in the tonometer by use of the $\mathrm{Lex}-\mathrm{O}_{2}$-Con (9).

The percentage of adult and fetal hemoglobin was obtained by 

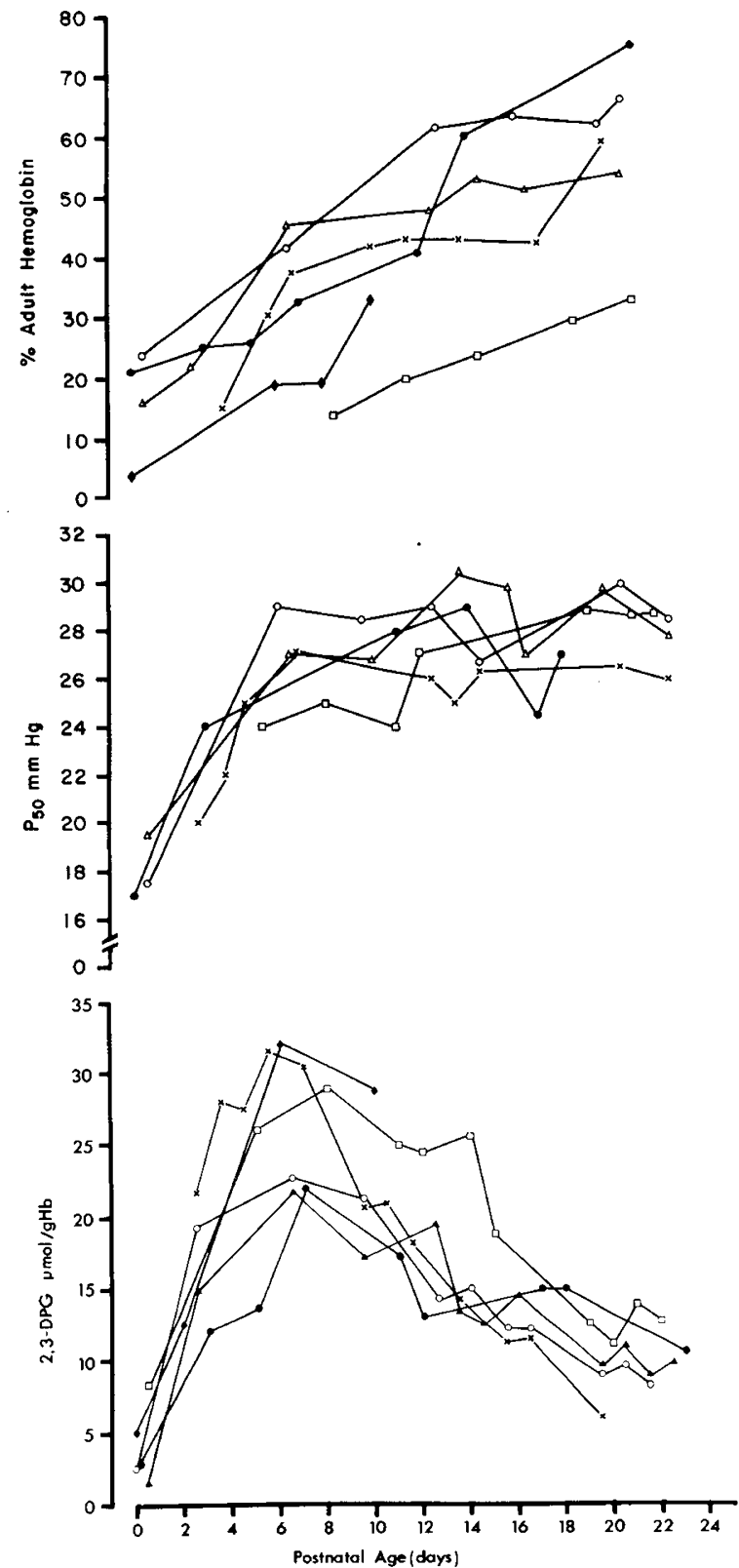

Fig. 1. The sequential changes of the percentage of adult hemoglobin (top), $\mathrm{P}_{50}$ (middle), and 2,3-diphosphoglycerate (bottom), are shown in relation to postnatal age in normal newborn lambs. The lines uniting the symbols represent repeated sampling of the same animal for the three parameters.

use of DEAE-Sephadex A-50 chromatography as described by Dozy et al. (3)

Six newborn lambs whose adult hemoglobin was of the BB type were included in the first part of the study (one of the lambs did not have serial $\mathrm{P}_{50}$ values taken).

The second part of the study consisted of performing an exchange transfusion on five newborn lambs during the first day after birth and then repeating the same determinations in the exact manner as was done in the first part of the experiment. The exchanges were equivalent to $1-2$ times the estimated newborn blood volume ( $8 \%$ of body weight). The hematocrit of the donor blood was increased to the level of that of the newborn by the removal of some of the plasma.

Significant limits for correlation were determined by calculation of the correlation coefficients. The results are expressed as mean values \pm standard deviation.

\section{RESULTS}

The sequential postnatal rise and fall of erythrocyte DPG, the percentage of adult and fetal hemoglobin, and changes in $P_{50}$ are illustrated in Figure 1. At less than $24 \mathrm{hr}$ of age the mean value of DPG was $2.82 \pm 1.2$. There was then a rapid rise which reached a mean maximum level of $26.71 \pm 4.98$ at 7.5 days $( \pm 1.1)$. Once the peak was attained there was a rapid decline and at the end of the study period (20-24 days) the mean value was $9.63 \pm 3.73$.

The simultaneous studies of whole blood oxygen dissociation curves demonstrated that at less than $24 \mathrm{hr}$ of age there was a mean $P_{50}$ of $18.0 \pm 1.1$. When the maximum level of DPG was reached, the average $P_{50}$ was $27.0 \pm 1.39$ and at the end of the study period the mean $\mathrm{P}_{50}$ had reached $28.2 \pm 1.6$.

Also noted was an increase in adult hemoglobin in relation to postnatal age in the same group of animals. During the first extrauterine day, the percentage of adult hemoglobin was $16.7 \pm$ 2.5. At the time of the maximum DPG concentration the mean percentage of adult hemoglobin was $31.1+11.1$. By the end of the study the values had increased to $60.8 \pm 15.3 \%$.

On the day the maximum level of DPG was reached, there was a statistically significant inverse correlation between the amount of adult hemoglobin and the quantity of DPG $(P<0.05, r=0.77)$. During the period when the DPG levels declined, its concentration was inversely related to the amount of adult hemoglobin $(r=0.68$, $P<0.001)$.

To confirm that there is an interaction of the type of hemoglobin and DPG levels postnatally, exchange transfusions were performed soon after birth $(13 \pm 6 \mathrm{hr})$ on five newborn lambs.

The effects of the exchange transfusion on $\mathbf{P}_{50}$ and DPG are shown on Figure 2. The exchange transfusion provided the newborn lambs with a lower hemoglobin oxygen affinity and the impressive rapid postnatal rise in DPG did not occur.

\section{DISCUSSION}

In sheep the fetal and adult hemoglobin values intrinsically have a wide difference on oxygen affinity; their adult red cells have very minimal amounts of DPG and maintain a low oxygen affinity compared with the fetal red cells. The postnatal rise of DPG in newborn lambs lowers the oxygen affinity of the red cells by decreasing the intraerythrocyte $\mathrm{pH}(1)$.

The newborn lamb, in order to rapidly switch from a high to low oxygen affinity before the amount of adult hemoglobin is adequate enough to provide the low affinity needed, makes use of the rapid rise of DPG as a compensatory mechanism for the presence of high affinity hemoglobin in the extrauterine environment.

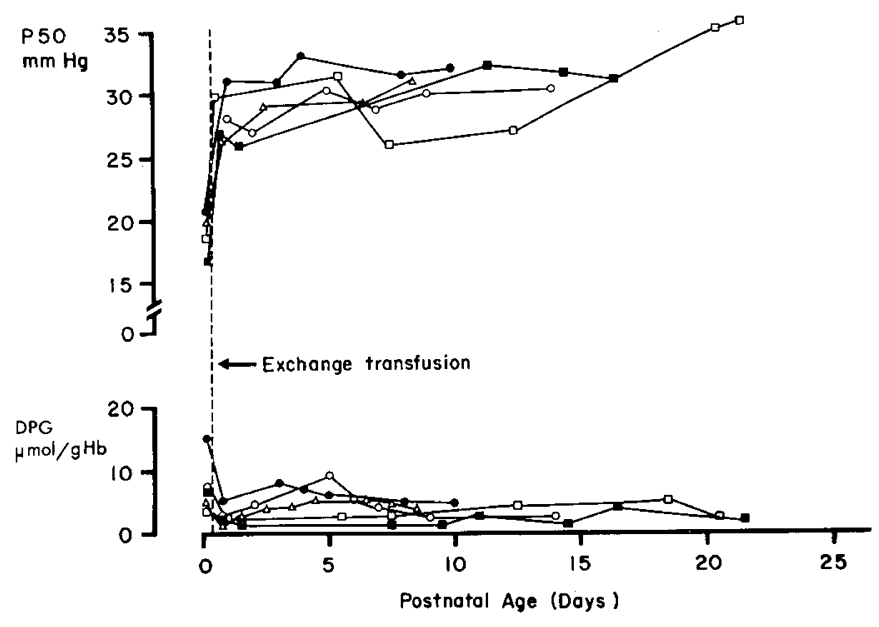

Fig. 2. The 2,3-diphosphoglycerate and $P_{50}$ in relation to postnatal age in five exchange transfused lambs. The lines uniting the different symbols represent sampling of the same animal. 


\section{SUMMARY}

This study demonstrates that the newborn lamb, in order to adapt to extrauterine life, requires erythrocytes with a lower oxygen affinity than that which existed near the end of fetal life. But because the red cells at birth contain predominantely fetal hemoglobin, an adequate $P_{50}$ can only be attained by a marked increase in red cell DPG. The amount of DPG required depends upon the quantity of adult hemoglobin present within the red cell at birth. When enough DPG has been attained for an adequate postnatal $\mathrm{P}_{50}$ the quantity then decreases synchronously with the increase in adult hemoglobin in an orderly fashion so that adequate $P_{50}$ values are maintained. The need for a rise in DPG concentration postnatally can be aborted by providing the newborn lamb with adequate amounts of adult hemoglobin, such as by an exchange transfusion with adult blood.

\section{REFERENCES AND NOTES}

I. Battaglia, F. C., McGaughey, H., Makowski, E. L., and Meschia, G.: Postnatal changes in oxygen affinity of sheep red cells: a dual role of diphosphoglyceric acid. Amer. J. Physiol., 219: 217 (1970).
2. Bunn, H. F., and Briehl, R. W.: The interaction of 2,3-diphosphoglycerate with various human hemoglobins. J. Clin. Invest., 49: 1088 (1970).

3. Dozy, A. M., Kleihauer, E. F., and Huisman, T. H. J.: Studies on the heterogeneity of hemoglobin. XIII. Chromatography of various human and animal hemoglobin types on DEAE-Sephadex. J. Chromatogr., 32: 723 (1968).

4. Hilpert, P., Fleischmann, R. C., Kempe, D., and Bartels, H.: The Bohr effect related to blood and erythrocyte pH. Amer. J. Physiol., 205(2): 337 (1963).

5. Keitt, A. S.: Reduced nicotinamide adenine dinucleotide-linked analysis of 2,3-diphosphoglyceric acid: Spectrophotometric and fluorometric procedures. J. Lab. Clin. Med., 77: 470 (1971).

6. Versmold, H., Seigert, G., and Riegel, K. P.: Blood oxygen affinity in infancy: The interaction of fetal and adult hemoglobin, oxygen capacity and red cell hydrogen ion and 2,3-diphosphoglycerate concentration. Resp. Physiol., 18: 14 (1973).

7. St. Louis, Mo.

8. Instrumentation Laboratory, Inc. Lexington, Mass.

9. Lexington Instruments Corp., Waltham, Mass.

10. The authors are grateful for the technical assistance provided by Mr. Andrew Bator, B.Sc.

11. This research was supported by Grant MA-5120 from the Medical Research Council of Canada.

12. Requests for reprints should be addressed to: H. Bard, M.D., Department of Pediatrics, Hôpital Sainte-Justine, 3175 Chemin Sainte-Catherine Montreal, Quebec H3T 1C5 (Canada).

13. Accepted for publication April 19, 1976.
Gas chromatography

D-glyceric acid

D-glyceric dehydrogenase hydroxypyruvic acid mass spectrometry nonketotic hyperglycinemia

\title{
D-Glyceric Acidemia: Biochemical Studies of a New Syndrome
}

\author{
S. K $\varnothing$ LVRAA, ${ }^{(32)}$ K. RASMUSSEN, AND N. J. BRANDT \\ Biochemical Research Unit, Department of Clinical Chemistry, University of Aarhus, Denmark and Department of \\ Teratology, University of Copenhagen, Rigshospitalet, Copenhagen, Denmark
}

\section{Extract}

Studies of a mentally retarded boy, clinically suffering from nonketotic hyperglycinemia, are reported. Using combined gas chromatography-mass spectrometry, enzyme specificity studies, and spectropolariometry D-glyceric acid in extremely elevated concentrations was demonstrated in both serum and urine (serum: 1.0-1.3 mmol/liter, urine: 33-187 mmol/liter). Hydroxypyruvic acid was not detectable in urine from this boy using a gas chromatographic method with a limit of detection of $0.3 \mathrm{mmol} /$ liter. Enzyme assays of D-glyceric dehydrogenase on blood leukocytes demonstrated significantly lower activity in the patient compared with five normal children.

\section{Speculation}

The accumulation of glycine in this new metabolic defect may result from a secondary inhibition of the glycine cleavage system, an explanation that would correspond well with the current concept on the genesis of the glycine accumulation found in ketotic hyperglycinemia.

Recently we had the opportunity to investigate a 4-year-old boy, clinically suffering from nonketotic hyperglycinemia, who con- stantly excreted large amounts of another compound besides glycine. This compound was identified as glyceric acid $(3,4)$.

Accumulation of this compound has not previously been reported in hyperglycinemic conditions. The original hyperglycinemia syndrome $(7,18)$, exists in two clinically distinct entities, a ketotic and a nonketotic form. The ketotic form is characterized by excretion of other metabolites besides glycine, i.e., organic acids derived from the breakdown of branched chain amino acids, and it is now firmly documented that also the primary enzyme defect is located here (16). The hyperglycinemia in these disorders is assumed to be a secondary phenomenon. In contrast, all so far available evidence indicates that nonketotic hyperglycinemia is caused by a primary defect in the glycine cleavage system, and it has never been possible to demonstrate accumulation of other metabolites besides glycine (17).

Hyperglyceric aciduria has previously been described only in primary hyperoxaluria type 2 , a condition characterized by recurrent nephrolithiasis and excretion of oxalic acid and L-glyceric acid $(25,26)$. However, hyperglycinemia is not a feature in this disorder.

The purpose of this paper is to present biochemical studies on our patient. It is shown that the glyceric acid excreted in this new metabolic disorder is the D-stereoisomer, and that the accumulation of this compound presumably results from an enzyme defect of the D-glyceric dehydrogenase. 